www.jmscr.igmpublication.org

Impact Factor 5.84

Index Copernicus Value: 83.27

ISSN (e)-2347-176x ISSN (p) 2455-0450

crossref DOI: _https://dx.doi.org/10.18535/jmscr/v5i3.83

Journal Of Medical Science And Clinical Research

IGM Publication

An official Publication of IGM Publication

\title{
Clinical Spectrum of Renal Dysfunction in Cirrhosis of Liver
}

\author{
Authors \\ Dr Twinkle Chandrakar ${ }^{1}$, Dr Tanuja Manohar ${ }^{2}$, Dr Nalini Humaney ${ }^{3}$ \\ ${ }^{1}$ Junior resident, ${ }^{2}$ Associate Professor, ${ }^{3}$ Professor \\ Department of Medicine, NKP Salve Institute of Medical Sciences and Research Centre \\ Lata Mangeshkar Hospital, Nagpur \\ Corresponding Author \\ Dr Twinkle Chandrakar \\ Lata Mangeshkar Hospital, Hingna road, Nagpur PIN-440019 \\ Email: twinklechandrakar9@gmail.com,Mobile Number-8806949490
}

\begin{abstract}
Introduction: Renal dysfunction is a common and serious problem in patients with advanced liver disease. Physicians involved in the care of patients of cirrhosis recognize that the development of renal dysfunction is associated with significant morbidity and mortality. Hence it is very important to diagnose condition early and recognize type of renal failure in order to decide further therapy.

Methods-This was a prospective single centered observational study was conducted on 100 patients with cirrhosis of liver admitted in a tertiary care hospital, according to inclusion and exclusion criteria during the study period Jan 2015 to Jul 2016. After collecting details of demographic data, subjects underwent complete general \& systemic examination and later subjected to blood investigations, ultrasonography abdomen and kub, urine examination and upper gastrointestinal endoscopy. Child-pugh score and MELD score was calculated.

Result- In this study, Renal dysfunction was present in $40 \%$ of population which was later categorized according to the type of renal involvement. $21 \%$ of total study population was identified to develop acute kidney injury, which was most common presentation for renal involvement and $18 \%$ were found to have hepatorenal syndrome. Only one (1\%) patient was diagnosed as chronic kidney disease. The most common etiology found for liver cirrhosis was alcoholic cirrhosis in both groups with or without renal dysfunction. In our study population total 16 patients were succumbed during hospitalization and all are belong to hepatorenal syndrome. Among the patients with fatal outcome maximum patients were male and had complications of spontaneous bacterial peritonitis, esophageal varices and were in CP class C with higher MELD score. Presence of spontaneous bacterial peritonitis and gastrointestinal bleed were major factors those were significantly associated with development of HRS. These factors alone or in combination were responsible for development of hepatorenal syndrome.

Conclusion-Renal dysfunction is a frequent and dreaded complication of cirrhosis of liver. An attempt should always be made to identify it early and categorize patients in different groups as treatment and prognosis differs in these subgroups. Patients usually have downhill course once hepato-renal syndrome develops while patients with AKI because of pre-renal azotemia can be successfully treated if detected early.
\end{abstract}




\section{Introduction}

Renal dysfunction is a common and serious problem In patients with advanced liver disease. Physicians involved in the care of patients of cirrhosis recognize that the development of renal dysfunction is associated with significant morbidity and mortality. Methods for early and accurate diagnosis of renal failure may assist to initiate specific treatment at earlier stage and improve the outcome. Patients with cirrhosis can commonly develop acute renal failure and may rarely suffer from underlying chronic kidney disease. Common types of renal dysfunction seen in cirrhosis of liver are hepatorenal syndrome; hypovolemia induced renal failure, renal failure because of intrinsic renal disease and druginduced renal failure. To pinpoint the specific type of renal failure in cirrhosis though difficult is important for both prognostic and therapeutic point of view. Once renal dysfunction sets in prognosis of patient worsens lead to progressive downhill course, leading to significant morbidity and mortality. Hence it is very important to diagnose condition early and recognize type of renal failure in order to decide further therapy. $1,2,3$.

Hepatorenal syndrome (HRS) is a unique form of acute kidney injury seen in patient with cirrhosis in the absence of any other identifiable cause of renal failure. It usually results from renal vasoconstriction in the setting of systemic and splanchnic arterial vasodilatation in patients with advanced cirrhosis. HRS is typically subdivided into two types: type-1 in which there is a rapid deterioration of kidney function with the serum creatinine increasing by more than $100 \%$ from baseline to greater than $2.5 \mathrm{mg} / \mathrm{dl}$ within a twoweek period, whereas type-2 HRS occurs in patients with refractory ascites with either a steady but moderate degree of functional renal failure $(\geq$ $1.5 \mathrm{mg} / \mathrm{dl}$ ) or a deterioration in kidney function that does not fulfill the criteria for HRS type-1. In patients with advanced cirrhosis, HRS is reported to occur in $18 \%$ within one year of diagnosis and up to $40 \%$ at five years. Untreated, median survival is two weeks for patients with type-1
HRS and four to six months in patients with type2 hepatorenal syndrome. ${ }^{4}$

Other types of renal dysfunction include 1 . hypovolemia induced renal failure which occurs in setting of excessive fluid loss as occurs in vigorous diuretic use, Gastro-intestinal bleeding, vomiting or diarrhoea. It can be treated effectively by restoring lost volume. 2.Intrinsic renal disease can also occur in cirrhosis as in hepatitis C. It can be detected by proteinuria > 500mg/ day, or hematuria micro or macroscopic and by altered echo structure in USG. Distinction between acute tubular necrosis and the hepatorenal syndrome 1 remains a difficult issue; the presence of renal tubular epithelial cells in the urine sediment favors the diagnosis of acute tubular necrosis. Current or recent treatment with nonsteroidal antiinflammatory drugs or aminoglycosides suggests drug-induced renal failure. ${ }^{5,6}$.

The term 'Hepatorenal Disorders' has been proposed to group all forms of kidney disease in patients with cirrhosis so as to describe their prognosis and to assist treatment decisions. ${ }^{7}$, in the majority of patients with advanced liver disease, HRS type-1 remains a terminal condition of requiring coordinated efforts for diagnosis and treatment.

Considering importance of renal dysfunction in natural history and prognostication, this project was undertaken to diagnose spectrum of renal dysfunction in cirrhosis and study risk factors for this dreaded complication. In our study, we are going to address the current knowledge on the diagnosis evaluation and risk assessment of renal dysfunction in patients with cirrhosis.

\section{Aims and Objectives}

1. To study prevalence of renal dysfunction in cirrhosis of liver.

2. To categorise patients with renal dysfunction into different types.

3. To study risk factors for development of hepatorenal syndrome.

4. To correlate severity of cirrhosis with occurrence of hepatorenal syndrome. 


\section{Materials and Methods Study Design}

This was a prospective single centered observational study was conducted on patients with cirrhosis of liver admitted in general medicine wards or intensive care unit, in a tertiary care hospital. Institutional Ethics Committee approval was obtained prior to commencement of the study. The data was collected after obtaining written informed consent from the patient.

Sample -100 consecutive of cirrhotic patients admitted during the study period 19 months (Jan 2015 to Jul 2016) as per the following inclusion/exclusion criteria were included in study.

\section{Inclusion criteria:}

- Patients above age 18 years

- Patients who were admitted in general medicine wards or intensive care unit with cirrhosis of liver and ascites.

- Patients willing to give written informed consent

\section{Exclusion Criteria:}

- Shock

- Severe sepsis

- Multi-organ failure

- Patient on maintenance hemodialysis

Shock is a clinical syndrome that result from inadequate tissue perfusion. Irrespective of cause, the hypoperfusion induced imbalance between the delivery of and requirements for oxygen and substrate leads to cellular dysfunction. Clinical shock is usually accompanied by hypotension (mean arterial pressure $<60 \mathrm{mmHg}$ in previously normotensive persons)

Severe sepsis is the harmful host response to infection systemic response to proven/suspected infection plus some degree of organ hypofunction arterial systolic blood pressure $\leq 90 \mathrm{~mm}$ of hg or mean arterial pressure $\leq 70 \mathrm{~mm}$ of hg that responds to administration of IV fluids. urine output 0.5 $\mathrm{ml} / \mathrm{kg}$ per hour for $1 \mathrm{hr}$ despite adequate fluid resuscitation. Pao2/Fio $2 \leq 250$ or, if the lung is the only dysfunctional organ $\leq 200$.platlet count < $80000 / \mu 1$ or $50 \%$ decrease in platlet count from highest value recorded over previous 3 days. A ph $<7.3$ or a base deficit $5.0 \mathrm{mEq} / 1$ and a plasma lactate level 1.5 times upper limit of normal for reporting lab.

Multiorgan failure is defined by simultaneous presence of physiologic dysfunction and/or failure of two or more organs. Typically, this syndrome occurs in the setting of severe sepsis, shock of any kind, severe inflammatory conditions.

\section{Data Collection}

Purpose of study was explained to the patient and written informed consent was obtained.

Data was obtained from the medical history and clinical examination and relevent investigation were in Case Record Form (CRF).

The following parameters were noted:

1) Patients Demographic Data: Name, Age, and Gender.

2) Detailed medical history for following variables was evaluated: Abdominal distention, pain in abdomen, fever, reduced urinary output, haematemesis, melaena, vomiting, diarrhoea, swelling of feet yellow discolouration of eyes, altered level of conscious, smoking, details of alcohol intake, hypertension, diabetes mellitus, chronic obstructive pulmonary disease, and ischemic heart disease

3) Clinical examination: Temperature, pulse, blood pressure, presence of pallor, icterus, clubbing, pedal odema, spider naevi, gynaceomastia, breast atrophy, loss of axillary hair, flapping tremor, palmar erythema, drupatryons contracture, testiucular atrophy, spleenomegaly, ascites, abdominal tenderness, crepts, consicousness, encephalopathy.

4) Laboratory Investigations:

- All patients were subjected to lab investigations in the form of Complete blood count, coagulation profile, Liver function test and Kidney function test.

- Appropriate cultures were sent in case of suspected infection. 
- Urinary sodium and urine osmolality were also estimated.

- Abdominal paracentesis was done and the ascitic fluid was subjected to biochemical and pathological examination in every patient

5. Radiological Investigations: All patient underwent Ultrasonography examination for evaluation of presence of ascites, examination of liver, spleen and kidneys.

Cirrhosis was diagnosed by clinical, biochemical and ultra-sonographic examination. Based on the patient details and investigations we also tried to find out etiology of cirrhosis and cryptogenic cirrhosis (unknown etiology) labelled after ruling out other common cause of cirrhosis of liver (like wilson disease, hepatitis B and C, autoimmune, drug induced). Patients were assessed for presence of renal dysfunction. Patients showing signs and findings related to renal dysfunction in presence of cirrhosis were identified, and a detailed evaluation was performed. Based on the clinical findings, biochemical parameters and radiological investigations, these patients were subcategorized as below categorizes.

\section{Based on acuity of presentation}

- Acute kidney injury

- Chronic kidney disease

- Acute on chronic kidney disease

\section{Based on the cause of renal dysfunction}

- Prerenal azotemia

- Hepatorenal syndrome

- Intrinsic renal disease: Acute tubular necrosis (ATN), glomerulopathies, acute interstitial nephritis (AIN), contrastinduced nephropathy and others

- Post-renal disease.

As follow up of patient were not done,patients could not be categarized between HRS1 and HRS2.

Patients with serum creatinine more than 1.5 $\mathrm{mg} / \mathrm{dl}$ were given albumin $1 \mathrm{gm} / \mathrm{kg}$ of body weight per day for 2 days with diuretic withdrawal. After 2 days if serum creatinine improve to less than or equal to $1.5 \mathrm{mg} / \mathrm{dl}$ they were labelled as acute kidney injury (AKI) because of prerenal azotemia and if no improvement was seen in serum creatinine they were labelled as hepatorenal syndrome $^{72}$

Hepatorenal syndrome was defined as per the International Ascites Club (IAC) definition.

Prerenal azotemia was defined using clinical and laboratory parameters. Urinary markers necessary for diagnosis included a urine specific gravity greater than 1.020 as well as a fractional excretion of sodium less than 0.01 (1\%) in oliguric patients not treated with diuretics. Diagnosis of Intrinsic renal disease required the presence of granular casts (epithelial cell casts) in urinary sediment, fractional excretion of sodium greater than $2 \%$, or a urinary sodium concentration greater than 40 $\mathrm{mmol} / \mathrm{L}$, in the clinical setting of prolonged hypotension, tachycardia, sepsis, or bleeding.

All the patients include in study were divided into two groups, those with renal dysfunction or without renal dysfunction. clinical parameter and laboratory parameter and frequency of complications, severity of the disease (by MELD and CPT score) and mortality were compared between two groups and statistically analyzed.

Score Calculation:

For assessment of severity liver dysfunction CTP score and MELD scores were calculated in all patients.

1) CTP:(Child-Turcotte-Pugh) Score- showen in Table no. 5 and 6,Fig. no. 3 and 4)

2) MELD: (Model for End-Stage Liver Disease) Score-showen in Table no.6a, Fig.no. 5 and 6)

\section{Statistical analysis of Data}

The statistical analysis of the data was performed using statistical package for social sciences (SPSS) software version 15.0. Demographics, clinical and laboratory characteristics of the patients were summarized using descriptive statistics like mean, standard deviation, for continuous variables and frequency and percentage for categorical variables. Categorical variables were compared using Chi-square test. Independent 
sample t test used to compare continuous variables in two groups. $\mathrm{P}$ value $<0.05$ was considered as significant.

\section{Results}

Table 10: Frequency and type of renal dysfunction in study population

\begin{tabular}{|l|c|}
\hline Renal Dysfunction & Frequency (\%) \\
\hline Present & $40(40.0)$ \\
\hline Absent & $60(60.0)$ \\
\hline Type of Renal Dysfunction & \\
\hline Acute Kidney Injury & $21(21 \%)$ \\
\hline Hepato-renal Syndrome & $18(18 \%)$ \\
\hline Chronic kidney disease & $1(1 \%)$ \\
\hline
\end{tabular}

Figure 8: Type of renal dysfunction in study population

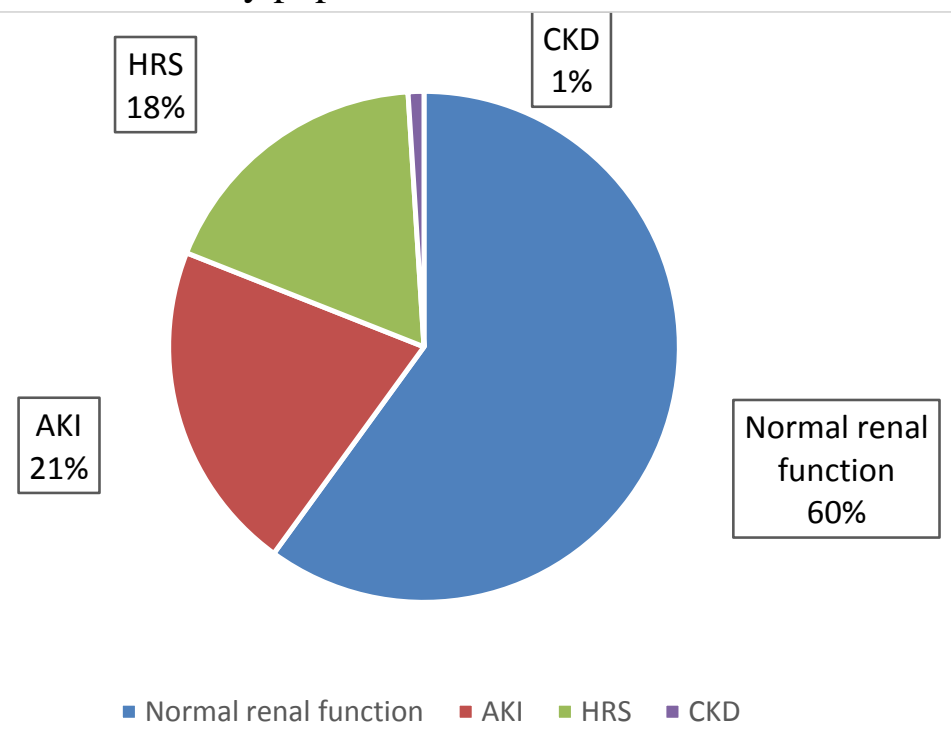

Renal dysfunction was present in $40 \%$ case in our study as shown in table 10/figure8. Amongst these syndrome, and chronic kidney disease patients, $21 \%, 18 \%$ and $1 \%$ had AKI, hepato-renal respectively.

Table 24: Child-Pugh classification of in both groups

\begin{tabular}{|l|c|c|c|}
\hline \multirow{2}{*}{ Child-Pugh Class } & \multicolumn{2}{|c|}{ Renal Dysfunction } & \multirow{2}{*}{ P values } \\
\cline { 2 - 3 } & Present $(\mathrm{n}=40)$ & Absent $(\mathrm{n}=60)$ & \multirow{2}{*}{0.009} \\
\hline B & $14(35 \%)$ & $37(61.7)$ & \\
\hline
\end{tabular}

Significantly higher percentage of patients with renal dysfunction $(65 \%)$ had more severe disease

Child-Pugh class (C) compared to those without renal dysfunction $(38.3 \%)$.

Table 25: Child-Pugh classification of in HRS cases

\begin{tabular}{|l|c|c|c|}
\hline Child-Pugh Class & $\begin{array}{c}\text { HRS-Present } \\
(\mathrm{n}=18)\end{array}$ & $\begin{array}{c}\text { HRS-Absent } \\
(\mathrm{n}=82)\end{array}$ & P values \\
\hline B & $4(22.2)$ & $47(57.3)$ & \multirow{2}{*}{0.007} \\
\hline C & $14(77.8)$ & $35(42.7)$ & \\
\hline
\end{tabular}

From among patients with HRS, over 3/4th was significantly higher than those without HRS patients had hepatic disease of CP class $\mathrm{C}$ which $(42.7 \%)(\mathrm{p}=0.007)$ as shown in table 25 . 
Table 26: MELD score distribution in both groups

\begin{tabular}{|l|c|c|c|}
\hline \multirow{2}{*}{ MELD Score } & \multicolumn{2}{|c|}{ Renal Dysfunction } & \multirow{2}{*}{ P values } \\
\cline { 2 - 3 } & Present $(\mathrm{n}=40)$ & Absent $(\mathrm{n}=60)$ & $<0.0001$ \\
\hline Mean & $26.0 \pm 8.6$ & $16.4 \pm 6.7$ & \\
\hline Categories according to the score & $3(5.0)$ & \multirow{2}{*}{$<0.0001$} \\
\hline 40 or more & $3(7.5)$ & 0 & \\
\hline $30-39$ & $8(20.0)$ & $3(5.0)$ & \\
\hline $20-29$ & $19(47.5)$ & $54(90.0)$ & \\
\hline $10-19$ & $10(25.0)$ & 0 & \\
\hline$<9$ & 0 & & \\
\hline
\end{tabular}

MELD score distribution is depicted in table 26.

Patients who had renal dysfunction were found to have significantly higher mean MELD score than those without renal dysfunction $(\mathrm{p}<0.0001)$. Also, proportion of patients with higher MELD score was significantly greater in patients with renal dysfunction $(\mathrm{p}<0.0001)$

Table 27: MELD score distribution in HRS population

\begin{tabular}{|l|c|c|c|}
\hline \multirow{2}{*}{ MELD Score } & \multicolumn{2}{|c|}{ HRS } & \multirow{2}{*}{ P values } \\
\cline { 2 - 3 } & Present $(\mathrm{n}-18)$ & Absent $(\mathrm{n}=82)$ & $<0.0001$ \\
\hline Mean & $33.1 \pm 7.7$ & $17.4 \pm 6.2$ & \multirow{2}{*}{$<0.0001$} \\
\hline Categories according to the score & $3(3.7)$ & \\
\hline 40 or more & $3(16.7)$ & 0 & \\
\hline $30-39$ & $8(44.4)$ & $16(19.5)$ & \\
\hline $20-29$ & $6(33.3)$ & $63(76.8)$ & 0 \\
\hline $10-19$ & $1(5.6)$ & 0 & 0 \\
\hline$<9$ & 0 & & \\
\hline
\end{tabular}

MELD score distribution is HRS cases depicted in table 27. Patients who had HRS were found to have significantly higher mean MELD score than those without HRS $(p<0.0001)$. Also, proportion of patients with higher MELD score was significantly greater in patients with HRS $(\mathrm{p}<0.0001)$.

Table 29: Distribution of important demographic, clinical and laboratory features in survivors and nonsurvivors

\begin{tabular}{|l|c|c|c|}
\hline \multirow{2}{*}{ variables } & \multicolumn{2}{|c|}{ Mortality } & \multirow{2}{*}{ P values } \\
\cline { 2 - 3 } & Yes $(\mathrm{n}=16)$ & No $(\mathrm{n}=84)$ & 0.141 \\
\hline Age & $45.9 \pm 5.7$ & $48.4 \pm 6.3$ & \multirow{2}{*}{0.019} \\
\hline Male & $13(81.3)$ & $81(96.4)$ & \multirow{2}{*}{0.616} \\
\hline History of Alcoholism & $3(18.7)$ & $3(3.6)$ & 0.001 \\
\hline Hematemesis & $15(93.8)$ & $81(96.4)$ & $<0.0001$ \\
\hline Melaena & $9(56.3)$ & $15(17.9)$ & $<0.0001$ \\
\hline Spontaneous bacterial peritonitis & $14(87.5)$ & $31(36.9)$ & 0.006 \\
\hline Esophageal varices & $15(93.8)$ & $16(19.0)$ & \multirow{2}{*}{0.005} \\
\hline CP Grade - B & $9(56.3)$ & $19(22.6)$ & $<0.0001$ \\
\hline CP Grade - C & $3(18.8)$ & $48(57.1)$ & $<0.0001$ \\
\hline MELD Score & $13(81.3)$ & $36(42.9)$ & $<0.0001$ \\
\hline Serum creatinine & $34.7 \pm 6.6$ & $17.5 \pm 6.1$ & $<0.0001$ \\
\hline Blood urea & $2.9 \pm 0.56$ & $1.5 \pm 0.71$ & $<0.0001$ \\
\hline Total bilirubin & $72.7 \pm 20.2$ & $53.1 \pm 14.3$ & $<0.0001$ \\
\hline SGOT & $16.6 \pm 9.3$ & $4.4 \pm 7.0$ & 0.009 \\
\hline SGPT & $222.2 \pm 159.3$ & $74.4 \pm 37.6$ & $<0.0001$ \\
\hline Total protein & $139.6 \pm 116.4$ & $43.5 \pm 15.9$ & $5.8 \pm 0.50$ \\
\hline INR & $5.4 \pm 0.52$ & $1.3 \pm 0.3$ & \\
\hline
\end{tabular}


Table 29 described the association of various population parameters with mortality outcome. Male gender $(p=0.019)$, hematemesis $(p=0.001)$, melaena ( $p<0.0001)$, SBP $(\mathrm{p}<0.0001)$, presence of oesophageal varices $(\mathrm{p}=0.006)$, severe hepatic disease $\mathrm{CP}$ class $\mathrm{C}(\mathrm{p}=0.005)$, higher MELD score, serum creatinine, blood urea, total bilirubin, SGPT/SGOT and INR ( $<<0.0001$ for all) and lower total protein levels $(\mathrm{p}=0.009)$ were associated with mortality.

Table 31: Risk factor for development of hepato-renal syndrome

\begin{tabular}{|l|c|c|c|}
\hline \multirow{2}{*}{ Risk factor } & \multicolumn{2}{|c|}{ HRS } & \multirow{2}{*}{ P value } \\
\cline { 2 - 3 } & Present $(\mathrm{n}=18)$ & Absent $(\mathrm{n}=82)$ & \\
\hline Sub-acute bacterial peritonitis (SBP) & $16(88.9)$ & $15(18.3)$ & $<0.0001$ \\
\hline GI bleed & $9(50.0)$ & $19(23.2)$ & 0.022 \\
\hline Hyponatremia & $13(72.2)$ & $55(67.1)$ & 0.672 \\
\hline Other - Infection (pneumonia) & $1(5.6)$ & $1(1.2)$ & 0.444 \\
\hline SBP with GI bleed & $8(44.4)$ & $6(7.3)$ & - \\
\hline GI bleed with hyponatremia & $8(44.4)$ & $12(14.6)$ & - \\
\hline SBP with hyponatremia & $11(61.1)$ & $8(9.8)$ & - \\
\hline SBP with GI bleed with hyponatremia & $7(38.9)$ & $2(2.4)$ & - \\
\hline
\end{tabular}

The risk factors associated with development of HRS are shown in table 31. Presence of SBP $(\mathrm{p}<0.0001)$, GI bleed $(\mathrm{p}=0.022)$ were major factors that were significantly associated with development of HRS. These factors alone or in combination were responsible for development of HRS as shown in table 32.

\section{Discussion}

This prospective, single-center observational study was undertaken to determine the prevalence of renal dysfunction in patients with cirrhosis of liver to categorize these patients into different types, determine proportion of patients with cirrhosis developing hepatorenal syndrome and evaluate the risk factors involved in causation of Hepatorenal syndrome.

Data was collected from 100 indoor patients with cirrhosis of liver with concomitant ascites.

In present study, renal dysfunction was found to be present in $40 \%$ of the patient with cirrhosis of liver. patients were further categorized according to the type of renal involvement. It was seen that acute kidney injury was present in $21 \%$ of population which was the most common presenting renal involvement, followed by hepatorenal syndrome which was present in $18 \%$ of patients. only one patient $(1 \%)$ was detected to have CKD in the entire population (Table10, Fig
8). The prevalence of $40 \%$ for renal dysfunction in our study was similar to another Indian study from North India $^{8}$ which showed that renal dysfunction were present in $44 \%$ patients with liver cirrhosis. Whereas, another Indian study from South India, showed that $22 \%$ patients with cirrhosis presented with renal dysfunction. ${ }^{9}$ Another study by Howard et al, found that out of total 93 patients with a diagnosis of liver cirrhosis and normal renal function at admission, 23 patients developed renal dysfunction later within 15 days of hospitalization. ${ }^{10}$

MELD score distribution was calculated and assessed. Patients who had renal dysfunction were found to have significantly higher mean MELD score than those without renal dysfunction $(p<0.0001)$. Similar result was seen in those with HRS ( $\mathrm{p}<0.0001)$. Also, proportion of patients with higher MELD score was significantly greater in patients with renal dysfunction $(\mathrm{p}<0.0001)$ and HRS ( $<<0.0001)$. Maximum of the patients had the score of MELD between the range of 20-29. In our study, 16 patients had mortality as an outcome during hospitalization, out of which all were identified to be in the HRS and renal dysfunction group. No patients with acute kidney injury or chronic kidney disease showed a fatal outcome. In our study, 16 patients had mortality as an outcome during hospitalization, out of which 
all were identified to be in the HRS and renal dysfunction group. No patients with acute kidney injury or chronic kidney disease showed a fatal outcome. Further we studied the distribution of important demographic, clinical and laboratory features in survivors and non-survivors. Our findings suggest that male population was more among the non-survivors. Among the patients with mortality outcome symptoms of hematemesis $(\mathrm{p}=0.001)$, melaena $(\mathrm{p}<0.0001)$ along with complications like sub-acute bacterial peritonitis $(p<0.0001)$, presence of oesophageal varices $(\mathrm{p}=0.006)$, severe hepatic disease child Pugh class C $\quad(p=0.005)$, higher MELD score, serum creatinine, blood urea, total bilirubin, SGPT/SGOT and INR ( $<<0.0001$ for all) and lower total protein levels $(\mathrm{p}=0.009)$ were associated with mortality. Damien et al in his study, determined outcome and mortality risk related to acute renal failure (ARF) in critically ill patients with cirrhosis. It was observed that cirrhotic patients with ARF had higher MELD scores at baseline that those without ARF. They had more respiratory failure and cardiovascular failure during ICU stay, longer stay in ICU, and a greater crude hospital mortality rate $(65 \%$ vs. $32 \%$ ). In case-control studies both mild and severe ARF were independently associated with mortality (HR, 2.6, and 4.2, respectively). Cirrhotic patients with mild ARF patients had a higher risk of death than those without ARF (relative risk, RR, 2.0). Severe ARF was associated with an increase matched risk of death (RR 2.6), higher mortality of $51 \%$, and higher risk-adjusted mortality rate $\left(2.1\right.$ vs. 0.9). ${ }^{11}$ Our study show similar findings on mortality suggesting a higher mortality in patients with renal dysfunction, depending on the severity of clinical presentation.

In our present study as well as in previous studies, patients with HRS generally had very high MELD scores of 20 or above. ${ }^{12}$ Such high MELD scores are associated with 3-month mortality rates of $75 \%$ or more, irrespective of the presence of renal failure. ${ }^{13}$ However, our data demonstrate that not only severity of disease as assessed by the MELD score but also the presence of HRS has independent prognostic relevance for patients with cirrhosis and renal failure. This confirms the results of a recently published very important paper by Allesandria et al. which conceivably demonstrated that, in addition to the MELD score, the presence of HRS is an independent predictor of survival in cirrhotic patients with HRS. The probability of survival after 3 months was only $10 \%$ in that study, which was significantly less than in patients with the same MELD score, but without HRS. In our present study, the 3-month survival for HRS 1 patients was 33\%. This slight discrepancy cannot be explained by different degrees of liver failure, as the MELD scores of HRS patients were almost similar (33.1 in our study vs 28 in the study described).

However nevertheless, serum creatinine which is used to analyze MELD score, should be interpreted with caution, since there is no universal standardized creatinine assay; there are interlaboratory variations, interactions with bilirubin and great influence by numerous nonrenal factors such as body weight, race, age, and gender. $^{14}$

Our study showed that esophageal varices (66.7\%), use of diuretics (47.6\%) and presence of diarrhoea and vomiting (23.8\% each) were major cause of acute kidney injury alone or in combination. Whereas along with this the risk factors for development of hepatorenal syndrome was studied which showed that presence of subacute bacterial peritonitis (88 \% vs $18.3 \%$, $\mathrm{p}<0.0001)$, GI bleed (50\% vs $23.2 \%, \mathrm{p}=0.022)$ were major factors that were significantly associated with development of HRS. Hyponatremia was also a common risk factor in both the groups but the difference was not statistically significant $(72.2 \%$ vs $67.1 \%$, $\mathrm{p}=0.672$ ). Other risk factors encountered were infections.

Our findings go with those reported by others. Follo et al. ${ }^{15}$ concluded that renal dysfunction is frequent in cirrhotic with spontaneous bacterial 
peritonitis that occurs mainly in patients with kidney failure before infection and found that renal failure is an important predictor of survival especially in the presence of SBP. Also, the same conclusion was mentioned in the study of Ruiz-del Arbol et al. ${ }^{16}$ as patients with SBP frequently develop a progressive severe renal and hepatic failure, and even death, despite of the resolution of infection and is usually associated with an extremely poor prognosis. These observations give an alarm for the emergency-pattern of combating coexisted infections in patients with ESLD to prevent these comorbidities and poor prognosis.

In our study we aimed to categorize the clinical presentation of renal dysfunction, the subtypes of renal involvement, hepatorenal syndrome along with assessment of risk factors and mortality. Martín-Llahí et al. ${ }^{(14)}$ discussed this drawback by the absence of specific markers that may help in the identification of the cause of renal failure in cirrhosis. We also recommend the need to exert an effort to build-up a new battery of laboratory markers of renal failure that may be useful for the differential diagnosis. The main limitation in our study was the short duration of the study and the lack of incorporating new adjuvant markers that may help in better differentiation of the possible etiology of renal failure in cirrhotic patients. Therefore, we recommend to investigate new tools for early detection and good delineation of renal dysfunction among cirrhotic that will reflect on prognosis.

\section{Conclusion}

Renal dysfunction is a frequent and dreaded complication of cirrhosis of liver. An attempt should always be made to identify it early and categorize patients in different groups as treatment and prognosis differs in these subgroups. Patients usually have downhill course once hepato-renal syndrome develops while patients with AKI because of pre-renal azotemia can be successfully treated if detected early. Patients with higher MELD score and Child $\mathrm{C}$ class patients are more likely to develop renal dysfunction especially hepatorenal syndrome. Bleeding esophageal varices, SBP and hepatic encephalopathy are important risk factors for development of renal dysfunction.. Early diagnosis, prevention and appropriate treatment of renal dysfunction can reduce the morbidity and mortality.

\section{References}

1. Cholongitas E, Senzolo M, Patch D, Shaw S, O'Beirne J, Burroughs AK. Cirrhotics admitted to intensive care unit: the impact of acute renal failure on mortality. Eur $\mathbf{J}$ Gastroenterol Hepatol. 2009; 21:744-750.

2. Garcia-Tsao G, Parikh CR, Viola A. Acute kidney injury in cirrhosis. Hepatology. 2008; 48:2064-2077.

3. Ginès $\mathrm{P}$, Schrier RW. Renal failure in cirrhosis. N Engl J Med. 2009; 361:12791290.

4. Moreau R, Lebrec D. Acute renal failure in patients with cirrhosis: perspectives in the age of MELD. Hepatology 2003;37:233243.

5. Barretti P, Soares VA. Acute renal failure: clinical outcome and causes of death. Ren Fail. 1997 Mar;19(2):253-257.

6. Elizabeth J. Lechner and Michael G. Risbano. J.B. Renal Complications In, Non-Pulmonary Complications of Critical Care: A Clinical Guide, Respiratory Medicine: Humana Press New York 2014; 1941.

7. Nadim K, Kellum A, Davenport A, Wong F, Davis C, Pannu N et al. Hepatorenal syndrome: the 8th International Consensus Conference of the Acute Dialysis Quality Initiative (ADQI) Group. Crit Care. 2012; 16:R23.

8. Prakash J, Mahapatra AK, Ghosh B, Arora $\mathrm{P}$, Jain AK. Clinical spectrum of renal disorders in patients with cirrhosis of liver. Ren Fail 2011; 33:40-6

9. Jaiganesh M, Krishnasamy N, Senthilkumar $\mathrm{R}$ and Sathish K. 
International Journal of Biomedical Research, Clinical profile of renal dysfunction in cirrhotic liver, 2016; 7(2): 073-076.

10. Howard H, Gaither B, Eleana Z, Hashem B. Risk factors for the development of renal dysfunction in hospitalized patients with cirrhosis. The American Journal of Gastroenterology (2001) 96, 2206-2210

11. Damien C, Bruno B, Jean-Jacques P, Michel R, Pierre C. The attributable mortality of acute renal failure in critically ill patients with liver cirrhosis. Intensive Medicine care. December 2005, Volume 31, Issue 12, pp 1693-1699.

12. Alessandria C, Ozdogan O, Guevara M, Restuccia T, Jiménez W, Arroyo V et al. MELD score and clinical type predict prognosis in hepatorenal syndrome: Relevance to liver transplantation. Hepatology. 2005;41(6):1282-1289.

13. Testro Angus P. Targeting circulatory dysfunction in cirrhosis: Terlipressin and the hepatorenal syndrome. Journal of Gastroenterology and Hepatology. 2009;24(11):1707-1709.
14. Pipili C, Cholongitas E. Renal dysfunction in patients with cirrhosis: Where do we stand? World Journal of Gastrointestinal Pharmacology and Therapeutics. 2014;5(3):156-168.

15. Follo A, Llovet J, Navasa M, Planas R, Forns X, Francitorra A et al. Renal impairment after spontaneous bacterial peritonitis in cirrhosis: Incidence, clinical course, predictive factors and prognosis. Hepatology. 1994;20(6):1495-1501.

16. Lenz K, Kapral C, Gegenhuber A, Buder R, Wewalka F. Systemic, renal, and hepatic hemodynamic derangement in cirrhotic patients with spontaneous bacterial peritonitis. Hepatology. 2004; 39(3):865-866. 